\title{
SINGLE-BUNCH INSTABILITY DRIVEN BY THE ELECTRON CLOUD EFFECT IN THE POSITRON DAMPING RING OF THE INTERNATIONAL LINEAR COLLIDER*
}

\author{
M. Pivi\#, T. Raubenheimer, SLAC, Menlo Park, CA 94025, USA; A. Ghalam, USC, USA \\ K. Harkay, ANL, USA; K. Ohmi, KEK, Japan; R. Wanzenberg, DESY, Germany \\ A. Wolski, LBNL, Berkeley, CA 94720, USA; F. Zimmermann, CERN, Geneva, Switzerland
}

\section{Abstract}

Collective instabilities caused by the formation of an electron cloud (EC) are a potential limitation to the performances of the damping rings for a future linear collider. In this paper, we present recent simulation results for the electron cloud build-up in damping rings of different circumferences and discuss the single-bunch instabilities driven by the electron cloud.

\section{INTRODUCTION}

In the positron damping ring (DR) of the International Linear Collider (ILC), an electron cloud may generate and reach high density. In the present design, the DR has a circumference of $17 \mathrm{~km}$, and the international collaboration is investing considerable effort to studying alternative configurations with reduced circumference. Build-up of the electron cloud is strongly dependent on the bunch separation, which scales with the DR circumference. Reducing the circumference of the positron DR might then make electron cloud effects much more severe.

In this paper, we discuss the thresholds for the head-tail instability driven by the electron cloud in damping rings of circumference $17 \mathrm{~km}, 6 \mathrm{~km}$ and $3 \mathrm{~km}$, with parameters shown in Table 1. Detailed reports of EC simulations for the $17 \mathrm{~km}$ DR are given in references [1,2,3]. Reports for the $6 \mathrm{~km}$ and $3 \mathrm{~km}$ options are also in preparation [4].

\section{SIMULATIONS SINGLE-BUNCH}

Description of the single-bunch simulation codes HEAD-TAIL, PEHTS can be found in $[1,5]$. The simulations presented in this paper include 30 or 40 beamcloud interaction points every turn.

The codes can model the electron cloud in a field-free region or in a dipole region. The simulation results representing the $17 \mathrm{~km}$ ring as an extended field free region using PEHTS are shown in Fig. 1.

The results for the various damping ring options using HEAD-TAIL are shown in Figs. 2-4 for a ring modeled as an extended strong dipole field region; numerical values for these cases are given in Table 2.

Typically, if an electron cloud forms, it evolves until its density reaches a level close to the charge neutralization.

The cloud density thresholds that give rise to a singlebunch instability in dipoles are compared with the average neutralization densities in Table 2.

\footnotetext{
*Work supported by the Director, Office of Science, High Energy Physics, U.S. DOE under Contract No. DE-AC02-76SF00515. \#mpivi@slac.stanford.edu
}

Table 1: Parameters for possible ILC damping rings.

\begin{tabular}{|l|c|c|c|}
\hline Circumference $[\mathrm{m}]$ & 17000 & 6114 & 3067 \\
\hline Energy $[\mathrm{GeV}]$ & 5.0 & 5.0 & 5.0 \\
\hline Bunch charge $\left[10^{10}\right]$ & 2.0 & 2.0 & 2.0 \\
\hline Horizontal tune & 76.31 & 56.58 & 51.28 \\
\hline Vertical tune & 41.18 & 41.62 & 31.59 \\
\hline Beam sizes $\sigma_{x, y}[\mu \mathrm{m}]$ & $103,7.3$ & $98,6.8$ & $76,5.5$ \\
\hline Mom. comp. $\left[10^{-4}\right]$ & 1.22 & 1.42 & 2.68 \\
\hline Bunch length $[\mathrm{mm}]$ & 6.0 & 6.0 & 6.0 \\
\hline Energy spread $\left[10^{-3}\right]$ & 1.3 & 1.5 & 1.2 \\
\hline Synchrotron Tune & 0.07 & 0.034 & 0.026 \\
\hline
\end{tabular}

The single-bunch thresholds are a factor $\sim 5$ below the neutralization level. Chambers are considered round $\mathrm{r}=22 \mathrm{~mm}$ in all DRs arcs and $\mathrm{r}=50 \mathrm{~mm}$ in the $17 \mathrm{~km}$ DR long straight sections.

Fig. 5 shows results obtained by representing the ring with EC only in a wiggler section of length $540 \mathrm{~m}$. The wiggler sections occupy $3 \%$ of the $17 \mathrm{~km}$ ring. Therefore, the local density threshold for the wiggler section should be $\rho_{\mathrm{e}} \approx 6 \times 10^{12} \mathrm{~m}^{-3}$ estimated by the threshold for the dipole case multiplied by a factor of 30 . Expectations are in agreement with simulations, as shown in Fig.5.

The nonlinear wiggler field can potentially trap electrons. The development of the electron cloud in wigglers [2,3] is still under investigation [4].

Furthermore, when simulating the single-bunch effect in the DR $17 \mathrm{~km}$ ring as an extended field free region, the cloud threshold is found to be a factor 2-3 lower [1,2], compare Figs. 1 and 2. An electron cloud in the long straight sections with large chamber size, can be prevented with a surface secondary electron yield lower than $1.9[1,2,3]$, which represents a safe margin.

\section{SINGLE BUNCH INSTABILITY}

A short-range wake field arising from electron cloud may cause a single-bunch instability.

Table 2: Ring average neutralization levels and singlebunch (SB) instability EC density thresholds ( $\rho_{e}$ in units $10^{12} \mathrm{~m}^{-3}$ ) for various DR options. The simulated SB thresholds are given for a ring modeled as a dipole region.

\begin{tabular}{|l|c|c|c|}
\hline Circumference & $17 \mathrm{~km}$ & $6 \mathrm{~km}$ & $3 \mathrm{~km}$ \\
\hline Neutralization $\rho_{e}\left[10^{12} \mathrm{~m}^{-3}\right]$ & 0.8 & 6.0 & 15 \\
\hline Simulated $\rho_{e}$ in arcs $\delta_{\max }=1.4$ & 0.4 & 8.0 & 17 \\
\hline SB: $\rho_{e}$ threshold $\left[10^{12} \mathrm{~m}^{-3}\right]$ & 0.2 & 1.0 & 3.0 \\
\hline Neutral. / SB threshold & 4.0 & 6.0 & 5.0 \\
\hline
\end{tabular}




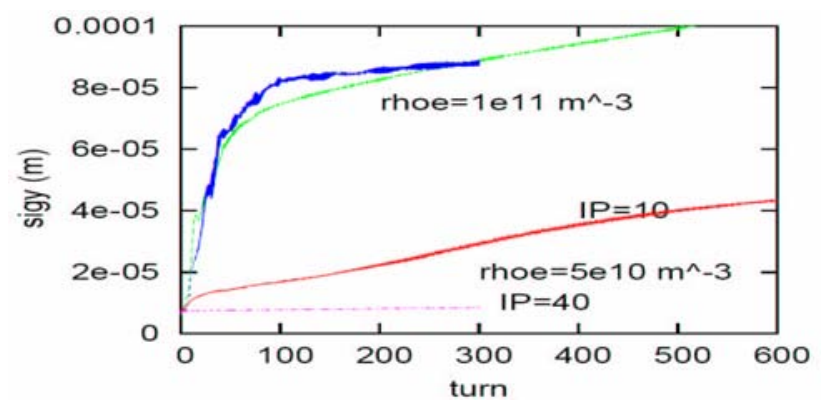

Figure 1. Evolution of the vertical beam size in the $17 \mathrm{~km}$ DR using PEHTS and an extended field free region representation. Results obtained for 10 or 40 beam-cloud interaction points.

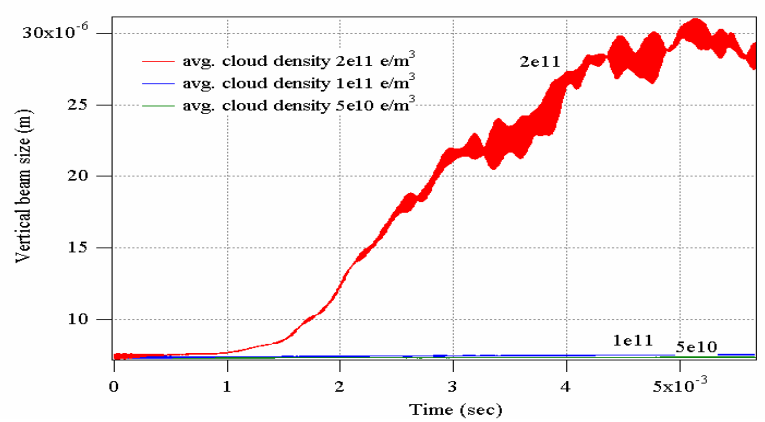

Figure 2. Evolution of the vertical beam size during 100 turns in the $17 \mathrm{~km}$ DR using HEAD-TAIL and a dipole extended model.

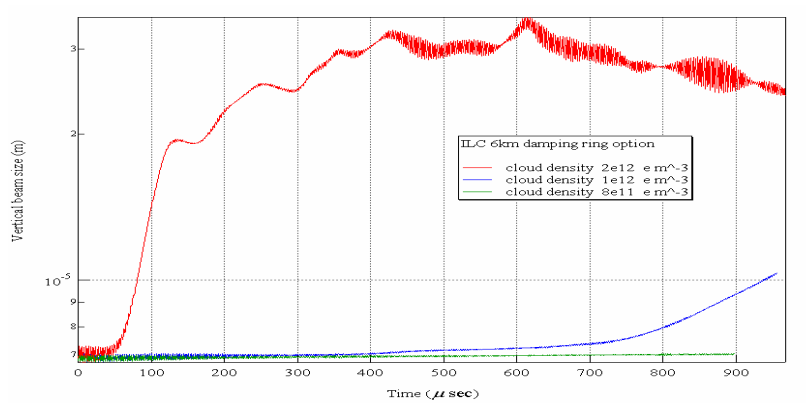

Figure 3. Vertical beam size evolution in the $6 \mathrm{~km}$ DR using a dipole extended model.

We use a resonator model [6,7] and focus on the vertical instability. The electron vertical oscillation frequency in the linearized beam potential is given by:

$$
\omega_{e, y}=\sqrt{\frac{\lambda_{+} r_{e} c^{2}}{\sigma_{y}\left(\sigma_{x}+\sigma_{y}\right)}}
$$

Where $\lambda+$ is the line density of particles in the beam and we consider a $2 \sigma_{\mathrm{z}}$ bunch length. The number of oscillations that an electron will make in one bunch passage is $\omega_{\mathrm{e}} \sigma_{z} / \mathrm{c}$, which is around 15 for all the lattices. It is therefore appropriate to analyze the instability using a coasting beam rather than a bunched beam model; the effect of the electron cloud in this case may be characterized as transverse microwave instability, rather than true head-tail instability.

In this case, we may estimate the impedance at which instability begins from:

$$
Z_{\text {th }}=4 \pi \frac{\gamma \alpha_{p} \sigma_{\delta} v_{y} a_{e} \sigma_{z}}{N_{0} r_{e} c^{2}}
$$

We assume that the wake field of the electron cloud can be represented in the frequency domain as an impedance corresponding to a resonator with resonant frequency $\omega_{e}$ :

$$
Z_{\perp}(\omega)=\frac{c R_{s}}{\omega} \frac{1}{1+i Q\left(\frac{\omega_{e}}{\omega}-\frac{\omega}{\omega_{e}}\right)}
$$

$R_{s}$ and $Q$ are parameters that characterize the shunt resistance and quality-factor of the resonator.

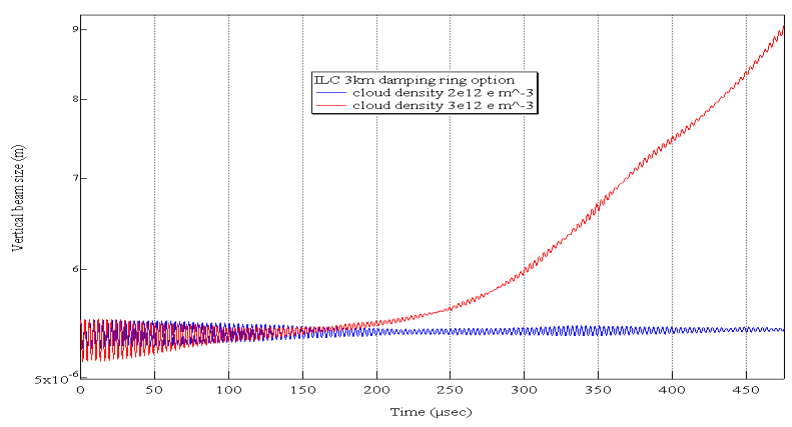

Figure 4. Vertical beam size evolution in the $3 \mathrm{~km}$ DR using a dipole extended model.

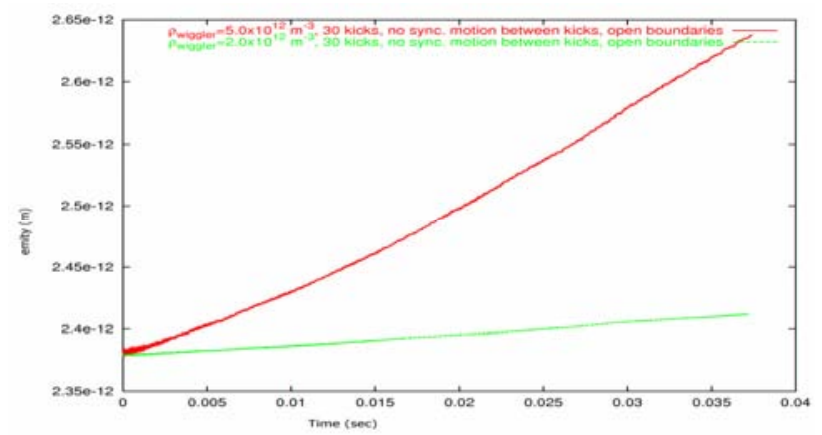

Figure 5. Evolution of the vertical emittance for different electron cloud density assuming the electron cloud occurs only in the $540 \mathrm{~m}$ long wiggler sections of the $17 \mathrm{~km}$ DR.

The imaginary part of the impedance has peak value (large $Q$ ):

$$
\hat{Z} \approx \frac{c R_{s}}{Q} \frac{Q}{2 \omega_{e}}
$$

The value of $c R_{s} / Q$ may be estimated from [7]:

$$
\frac{c R_{s}}{Q}=\frac{2 \pi \rho_{e} a_{e} C}{\lambda_{+} c}
$$

Using Eqs. (4) and (5) and assuming a value for $Q$, we may estimate the impedance of the electron cloud at the instability threshold, and compare with the analytical prediction from Eq. (2). The results are shown in Table 3. The threshold of the instability in the simulations is consistent with the predictions of the analytical model.

We may also determine the wake field from a simulation, using the following procedure. We model a coasting beam traversing an electron cloud represented by 100,000 macro-electrons, initially uniformly distributed. We displace a slice at the head of the bunch by $\sigma_{y}$ in the vertical direction. 
Table 3: Analytical estimate of single-bunch vertical threshold strong dipole field case.

\begin{tabular}{|l|c|c|c|}
\hline Circumference & $17 \mathrm{~km}$ & $6 \mathrm{~km}$ & $3 \mathrm{~km}$ \\
\hline$\rho_{e}\left[10^{12} \mathrm{~m}^{-3}\right]$ & 0.2 & 1.0 & 3.0 \\
\hline$c R_{S} / Q$ Eq. $(5)\left[10^{8} \mathrm{~m}^{-2}\right]$ & 0.34 & 0.66 & 1.2 \\
\hline$Q$ (assumed value) & 17 & 17 & 17 \\
\hline$\omega_{\mathrm{e}}$ Eq. $(1)\left[10^{11} \mathrm{~s}^{-1}\right]$ & 7.2 & 7.7 & 9.7 \\
\hline$\hat{Z}$ Eq. $(4)\left[10^{-4} \mathrm{~m}^{-2} \mathrm{~s}\right]$ & 4.0 & 7.3 & 10 \\
\hline$Z_{\text {th }}$ Eq. $(2)\left[10^{-4} \mathrm{~m}^{-2} \mathrm{~s}\right]$ & 6.1 & 9.0 & 13 \\
\hline
\end{tabular}

The subsequent 100 bunch slices are located on-axis and the wake field is computed from the force acting on the beam (using the Bassetti-Erskine formula). The vertical and horizontal wakes for the $17 \mathrm{~km}$ DR assuming electrons moving in a field free or strong dipole field are shown in Fig. 6 and 7 respectively for an extended cloud size $(1,1)$ and $(10,10)$ in units of $(\sigma x, \sigma y)$. Numerical results for all the DR options considered here are given in Table 4. Note that the wake field in Fig. 7, has factor $\mathrm{Q}=8$ and amplitude $3.4 \times 10^{8} \mathrm{~m}^{-2}$ at $\rho_{e}=10^{12} \mathrm{~m}^{-3}$, which is two orders of magnitude larger than that found for the KEK-B positron ring [6]. The simulated wake field parameters shown in Table 4 (in particular the values for $C R_{s} / Q$ ) are consistent with analytical estimates in Table 3; the frequencies are somewhat different because the simulation includes the full nonlinear forces, while the analytical estimate makes a linear approximation. We may again use Eq. (4) to calculate an effective impedance corresponding to the wake field: the impedances at the instability cloud density thresholds in the different damping rings calculated in this way are consistent with the analytical values in Table 3. The difference in the estimated impedance between Table 3 and 4 is due to the difference in frequency.

Consistently with estimations for other rings [8], the presence of the dipole field does not affect the simulated vertical wake field driven by the electron cloud but suppresses the horizontal wake field, as shown in Fig. 6 and 7. Nevertheless, the threshold of the instability in the vertical direction does depend on the presence of the dipole field, compare Figs. 1 and 2. We may conjecture as done in [8] - that the dipole field almost cancels any horizontal coherent motion and it also weakens the rather strong instability in the vertical direction.

\section{CONCLUSIONS}

We computed the single-bunch instability thresholds for the different DR options. The cloud density thresholds for the instability are a factor $\sim 5-10$ below the average neutralization levels. Both the cloud density and the single-bunch instability threshold increase with the reduction in length of a DR from the $17 \mathrm{~km}$ to the $3 \mathrm{~km}$, maintaining the ratio between average neutralization and SB threshold almost constant. It should be taken into account that in the $17 \mathrm{~km}$ DR long straight sections, the electron cloud may be prevented, in which case the single-bunch thresholds would be consequently higher.
Table 4: Simulation of vertical wake field for a strong dipole case, assuming cloud size $(1,1)$ in units of $(\sigma x, \sigma y)$. Wake amplitudes, see Fig. 6, are then computed at the listed threshold cloud density $\rho_{\mathrm{e}}$.

\begin{tabular}{|l|c|c|c|}
\hline Circumference & $17 \mathrm{~km}$ & $6 \mathrm{~km}$ & $3 \mathrm{~km}$ \\
\hline$\rho_{e}\left[10^{12} \mathrm{~m}^{-3}\right]$ & 0.2 & 1.0 & 3.0 \\
\hline$c R_{s} / Q\left[10^{8} \mathrm{~m}^{-2}\right]$ & 0.33 & 0.62 & 1.15 \\
\hline$Q$ & 17 & 17.1 & 17.1 \\
\hline$\omega_{R}\left[10^{11} \mathrm{~s}^{-1}\right]$ & 9.7 & 10.3 & 13 \\
\hline$\hat{Z}$ Eq. $(4)\left[10^{-4} \mathrm{~m}^{-2} \mathrm{~s}\right]$ & 2.9 & 5.2 & 7.5 \\
\hline
\end{tabular}

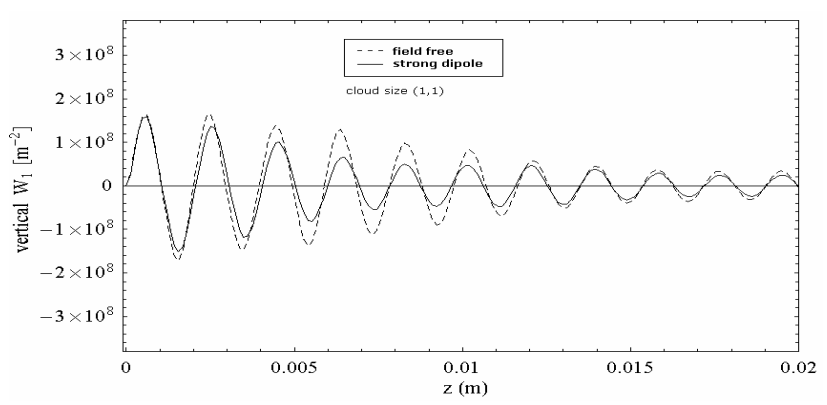

Figure 6. Vertical wake field computed for the DR $17 \mathrm{~km}$ field free region and strong dipole field. Wake computed with a cloud sizes $(\sigma \mathrm{x}, \sigma \mathrm{y})$ and cloud density $\rho_{e}=10^{12} \mathrm{~m}^{-3}$.
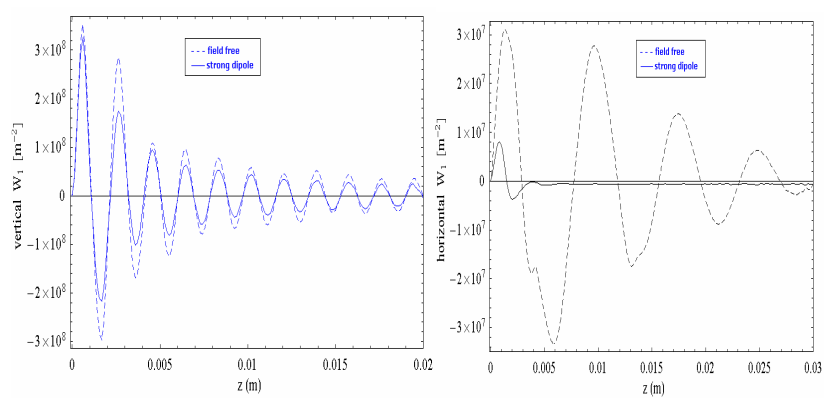

Figure 7. (Left) Vertical and (Right) Horizontal wake fields computed for the DR $17 \mathrm{~km}$ in field free and strong dipole field. Cloud size $(10,10)$ in units of $(\sigma x, \sigma y)$ and cloud density $\rho_{e}=10^{12} \mathrm{~m}^{-3}$.

The analytic estimates are in agreement with simulations. The instability is rather strong and the simulated wake fields are 1-2 orders of magnitude larger than what previously computed for other rings [7,8].

We would like to thank G. Rumolo, E. Benedetto, D. Shulte and M. Furman for very useful discussions.

\section{REFERENCES}

[1] K. Ohmi, proceedings of ECLOUD 2004.

[2] M. Pivi, proceedings of ECLOUD 2004.

[3] F.Zimmermann, R.Wanzenberg, D.Schulte,ECLOUD04.

[4] M. Pivi, F. Zimmermann, in preparation.

[5] G.Rumolo,F.Zimmeermann, PRST-AB 5,121002,2002

[6] K. Ohmi, F. Zimmermann, proceedings EPAC 2000.

[7] K. Ohmi et al., F. Zimmermann, E. Perevedentsev, Phys. Rev. E 65016502.

[8] L. Wang, G. Rumolo, K. Ohmi, F. Zimmermann, CERN-SL-2001-058 (AP). 